\title{
Proteomic Analysis of Rhesus Macaque Brain Explants Infected With Borrelia Burgdorferi Identifies Host GAP-43 As A Potential Factor Associated With Lyme Neuroborreliosis
}

Lianbao Li

Kunming Medical University

Lisha Luo

Kunming Medical University

Taigui Chen

Kunming Medical University

Wenjing Cao

Kunming Medical University

Xin Xu

Kunming Medical University

Yu Zhang

Kunming Medical University

Peng Yue

Kunming Medical University

\section{Yuxin Fan}

Kunming Medical University

Jingjing Chen

Kunming Medical University

Meixiao Liu

Kunming Medical University

Mingbiao Ma

Kunming Medical University

Lvyan Tao

Kunming Medical University

Yun Peng

Kunming Medical University

Yan Dong

Kunming Medical University

Bingxue Li

Kunming Medical University 


\section{Suyi Luo}

Kunming Medical University

\section{Jing Kong}

Kunming Medical University

\section{Guozhong Zhou}

Kunming Medical University

\section{Shiyuan Wen}

Kunming Medical University

\section{Aihua Liu}

Kunming Medical University

\section{Fukai Bao ( $\sim$ baofukai@126.com )}

Kunming Medical University https://orcid.org/0000-0003-2652-6660

\section{Research}

Keywords: Proteomic analysis, Lyme neuroborreliosis, Neuroinflammation, HMC3, Borrelia burgdorferi, GAP-43

Posted Date: December 16th, 2020

DOI: https://doi.org/10.21203/rs.3.rs-126769/v1

License: (c) (i) This work is licensed under a Creative Commons Attribution 4.0 International License. Read Full License 


\section{Abstract}

Background: Lyme neuroborreliosis (LNB) is one of the most dangerous manifestations of Lyme disease, but the pathogenesis and inflammatory mechanisms are not fully understood.

Methods: Cultured explants from the frontal cortex of rhesus monkey brain $(n=3)$ were treated with live Borrelia burgdorferi (Bb) or phosphate-buffered saline (PBS) for 6, 12, and $24 \mathrm{~h}$. Total protein was collected for sequencing and bioinformatics analysis. Changes in protein expression in the explants over time following $\mathrm{Bb}$ infection were screened.

Results: We identified 1237 differentially expressed proteins (DEPs; fold change $\geq 1.5$ or $\leq 0.67$, P-value $\leq 0.05$ ). One of these, growth-associated protein 43 (GAP-43), was highly expressed at all time points in the explants. The results of the protein-protein interaction network analysis of DEPs suggested that GAP43 plays a role in the neuroinflammation associated with LNB. In HMC3 cells incubated with live Bb or PBS for 6, 12, and $24 \mathrm{~h}$, real-time PCR and western blot analyses confirmed the upregulation of GAP-43 mRNA and protein, respectively.

Conclusions: Elevated GAP-43 expression is a potential marker for LNB that may be useful for diagnosis or treatment.

\section{Background}

Lyme disease (LD), a zoonosis that was first identified in the United States in the mid-1970s, is caused by the tick-borne bacterium Borrelia burgdorferi $(\mathrm{Bb})$; typical symptoms are multiphase and multisystem disorder [1]. Erythema migrans is a skin condition that is observed in the early stage of the disease. Without treatment, the infection can spread to the peripheral nervous system and central nervous system (CNS), leading to Lyme neuroborreliosis (LNB) [2, 3]. In 431 patients with LNB from Denmark, the main manifestations were painful radiculitis $(65.9 \%)$, cranial palsy (43.4\%; mostly facial palsy), and headache (28.3\%) [4]. Cytokines and chemokines released by immune cells-including those of the nervous system such as microglia (the brain-resident macrophages), astrocytes, and oligodendrocytes (the myelinating cells of the CNS) - have been linked to the neuropathogenesis of LNB [5]. The treatment of LNB is complicated by its multisystem etiology, and prevention and early treatment are critical to optimising outcomes [6].

Non-human primates (NHPs) are useful models for investigating human disease including LD, given their genetic and physiologic similarities and close phylogenetic relationship to humans [7]. The rhesus monkey is suitable for investigating the immunologic response to $\mathrm{Bb}$ infection in LNB [8] and is presently the only available LNB model. However, the use of NHPs for research is limited by ethical considerations and high cost; these can be circumvented by using human brain organoids as experimental models.

Microglia detect extracellular changes in the brain and are rapidly activated in response to various noxious stimuli $[9,10]$. Inappropriate microglia activation has been implicated in several neurologic 
diseases $[11,12]$. We speculated that microglia are involved in the pathogenesis of LNB. Proteomic approaches can be used for protein expression profiling in investigations on LD pathogenesis to identify differentially expressed proteins (DEPs) that can serve as biomarkers or potential drug targets [13-15]. In the present study, we used isobaric tags for relative and absolute quantitation (iTRAQ) proteomics technology to assess changes in protein abundance [16] in the brain upon spirochetes infection using frontal cortex (FC) explants from rhesus macaque and cultured cells infected with $\mathrm{Bb}$.

\section{Materials And Methods}

\section{Animals}

Three 1-year-old rhesus macaques (Macaca mulatta) of Chinese origin weighing 1.5-3 kg (Monkey-1 and -2 , male and Monkey-3, female) were obtained from the Institute of Medical Biology, Chinese Academy of Medical Sciences and Peking Union Medical College [animal permit no. SCXK (DIAN) K20150004]. The animals were healthy and were not infected with $\mathrm{Bb}$. We obtained FC tissue immediately after the animals were euthanised using $\mathrm{CO}_{2}$. This experiment was performed according to the Guide for the Care and Use of Laboratory Animals and ARRIVE Guidelines for Reporting Animal Research. The Animal Ethics and Welfare Committee of Kunming Medical University reviewed and approved this study.

\section{Preparation of live $\mathrm{Bb}$}

A 1-ml volume of Bb strain 4680 (Leibniz Institute DSMZ, Braunschweig, Germany; A1511358-1) frozen at $-80^{\circ} \mathrm{C}$ was added to $50 \mathrm{ml}$ of Barbour-Stoenner-Kelly II medium $[17,18]$ supplemented with $6 \%$ rabbit serum; the mixture was placed in a dark, tightly capped tube at $37^{\circ} \mathrm{C}$ for seven days. The supernatant was discarded after centrifugation $\left(4^{\circ} \mathrm{C}, 10 \mathrm{~min}, 2000 \times \mathrm{g}\right)$ when the cell count was $60-120$ per highpower field as determined by dark-field microscopy. After two washes with sterile phosphate-buffered saline (PBS), an appropriate volume of 10\% FBS-Roswell Park Memorial Institute (RPMI) 1640 medium with $10 \%$ fetal bovine serum (FBS) (Thermo Fisher Scientific, Waltham, MA, USA) for co-culture with FC explants or Minimal Essential Medium (MEM) (Shanghai Zhong Qiao Xin Zhou Biotechnology Co, Shanghai, China) for co-culture with HMC3 human microglia cells was added to a final bacterial concentration of $1 \times 10^{7} / \mathrm{ml}$.

\section{FC explant co-culture with live $\mathrm{Bb}$}

Fresh FC tissue from 3 rhesus macaques was washed twice with PBS and cut into extremely thin sections using a sterile scalpel [Jinzhong, Shanghai, China; J11060 (handle) and J0B080 (blade)] and atraumatic tissue forceps (Jinzhong; JYF010). Tissue fragments weighing $0.5 \mathrm{~g}$ were placed in separate T-25 flasks (Corning, Corning, NY, USA) containing $4 \mathrm{ml}$ of RPMI 1640 medium with 10\% FBS or $4 \mathrm{ml} \mathrm{Bb}$ suspension prepared as described above. We ensured that each tissue fragment was thoroughly permeated by the medium, and triplicate tissue fragments from the three animals were cultured in a humidified incubator at $37^{\circ} \mathrm{C}$ and $5 \% \mathrm{CO}_{2}$ for 6,12 , and $24 \mathrm{~h}$, after which they were collected and stored at $-80^{\circ} \mathrm{C}$ (Fig. 1). 


\section{iTRAQ-based proteomic analysis}

\section{Protein extraction and sample preparation}

$\mathrm{FC} / \mathrm{Bb}$ samples were added to protein lysis solution [7 M urea, $2 \mathrm{M}$ thiourea, $4 \%$ sodium dodecyl sulphate, $40 \mathrm{mM}$ Tris- $\mathrm{HCl}$ (pH 8.5), $1 \mathrm{mM}$ phenylmethane sulfonyl fluoride (PMSF), and $2 \mathrm{mM}$ EDTA] and incubated on ice for $5 \mathrm{~min}$. Dithiothreitol (DTT) was added to a final concentration of $10 \mathrm{mM}$, followed by ultrasonication for $15 \mathrm{~min}$ in ice. The supernatant was centrifuged $\left(4^{\circ} \mathrm{C}, 10 \mathrm{~min}, 13,000 \times \mathrm{g}\right)$ and cold acetone was added, followed by overnight incubation at $-20^{\circ} \mathrm{C}$.

The protein precipitate was collected by centrifugation and air-dried. After adding a solution of $8 \mathrm{M}$ urea and $100 \mathrm{mM}$ triethylammonium bicarbonate (TEAB) ( $\mathrm{pH} \mathrm{8.0)}$ to redissolve the proteins, DTT was added to a final concentration of $10 \mathrm{mM}$ and the reduction reaction was carried out in a water bath at $56^{\circ} \mathrm{C}$ for $30 \mathrm{~min}$. lodoacetamide was added at a final concentration of $55 \mathrm{mM}$ and the mixture was incubated at room temperature for $30 \mathrm{~min}$ in the dark for the alkylation reaction. The Bradford assay was used to determine the protein concentration, and $100 \mu \mathrm{g}$ of protein from each sample was used for trypsin digestion. After diluting the protein solution 1:4 with $100 \mathrm{mM}$ TEAB, trypsin was added at trypsin:protein mass ratio of $1: 50$ followed by enzymatic hydrolysis overnight at $37^{\circ} \mathrm{C}$. The resultant peptides were desalted and freeze-dried under vacuum.

iTRAQ labeling and mass spectrometry

iTRAQ-8 labelling was performed using a commercial kit (Sciex, Redwood City, CA, USA) according to the manufacturer's instructions. Briefly, peptides were dissolved in 0.5 M TEAB and labelled and mixed. The Ultimate 3000 high-performance liquid chromatography system (Dionex, Thermo Fisher Scientific, USA) was used for fractionation, and the 42 subfractions were combined into 12 samples that were desalted and vacuum-dried on a Strata-X column and dissolved in a solution of $2 \%$ acetonitrile and $0.1 \%$ formic acid. Mass spectrometry (MS) analysis was performed using a TripleTOF $5600+$ instrument coupled with the Eksigent nanoLC system (Sciex, USA).

The peptide solution was added to a C18 capture column $(5 \mu \mathrm{m}, 100 \mu \mathrm{m} \times 20 \mathrm{~mm})$, and gradient elution was performed on a C18 analytical column $(3 \mu \mathrm{m}, 75 \mu \mathrm{m} \times 150 \mathrm{~mm})$ with a mobile phases $\mathrm{A}(2 \%$ acetonitrile, $0.1 \%$ formic acid and $98 \% \mathrm{H}_{2} \mathrm{O}$ ) and a mobile phases $\mathrm{B}(98 \%$ acetonitrile, $0.1 \%$ formic acid and $2 \% \mathrm{H}_{2} \mathrm{O}$ ) at a flow rate of $300 \mathrm{nl} / \mathrm{min}$. The iTRAQ-based proteomics data were acquired using Information Dependent Acquisition (IDA), the MS spectrum was scanned with an ion accumulation time of $250 \mathrm{~ms}$ and $50 \mathrm{~ms}$ automated MS/MS product ion scans were performed for the top-30 ions with the highest intensity. IDA parameters were set as follows: collect MS1 spectrum in the range of $350-1500 \mathrm{~m} / \mathrm{z}$, collect MS2 spectrum in the range of $100-1500 \mathrm{~m} / \mathrm{z}$ and the dynamic exclusion time was $15 \mathrm{~s}$.

\section{Bioinformatics Analysis}


After optimisation processing, tandem MS data were analysed using the Proteinpilot v4.5 search engine, which is aligned to the TripleTOF $5600+$ system. Only peptides with unused score $\geq 1.3$ (reliability level > $95 \%$ ) were retained for protein quantification. DEPs were identified based on the ratios of differently labelled proteins and $P$ values in Proteinpilot. Fold change was calculated as an average value from paired comparisons among biological replicates. We set restrictive conditions in order to identify proteins involved in nervous system damage; only those with fold change in expression $\leq 0.67$ or $\geq 1.5$ and Pvalue $<0.05$ for differences in protein abundance in all pairwise comparisons were identified as DEPs. To determine the function and biological significance of identified proteins, a homology search was performed in the UniProt database for rhesus macaque (https://www.uniprot.org/), and homologous proteins were subjected to Gene Ontology (GO; http://geneontology.org/), Clusters of Orthologous Genes (COG; http://www.ncbi.nlm.nih.gov/COG/); and Kyoto Encyclopedia of Genes and Genomes (KEGG) pathway (https://www.kegg.jp/kegg/pathway.html) analyses. We also performed functional enrichment (GO and KEGG pathway) analyses for significantly different proteins.

\section{HMC3 cells co-culture with live Bb}

HMC3 cells (Shanghai Zhong Qiao Xin Zhou Biotechnology Co, Shanghai, China) were cultured in MEM with $5 \%$ cattle serum and $1 \%$ penicillin/streptomycin (Sangon Biotech, Shanghai, China) at $37{ }^{\circ} \mathrm{C}$ and $5 \%$ $\mathrm{CO}_{2}$. The cells were seeded in a 6-well plate at $2.5 \times 10^{5} \mathrm{cells} / \mathrm{ml}$. After $12 \mathrm{~h}$, the supernatant was discarded when the cells fully adhered to the plate. The cells were divided into two groups (negative control and experimental) that were stimulated with $2 \mathrm{ml} /$ well MEM and $1 \times 10^{7}$ cells $/ \mathrm{ml} \mathrm{Bb}$ in $0.25 \%$ PBS, respectively, for 6,12 , and $24 \mathrm{~h}$. Cell lysates were prepared using RNAiso Plus reagent (Takara Bio, Otsu, Japan) and stored at $-80^{\circ} \mathrm{C}$ until RNA extraction. Protein lysates were prepared using PMSF and radioimmunoprecipitation assay (RIPA) buffer (Solaibao Life Science, Beijing, China) and stored at $-80^{\circ} \mathrm{C}$ until western blot analysis (Fig. 1).

\section{RNA isolation and reverse transcription}

RNAiso Plus reagent (Takara Bio) was used to extract total RNA from $20 \mathrm{~g}$ of brain tissue; chloroformisopropanol extraction was performed to obtain RNA from HMC3 cells. After incubation for $30 \mathrm{~min}$, the samples were centrifuged and isolated RNA was washed with $75 \%$ ethanol. RNA concentration and purity were determined with a Nanodrop-2000 spectrophotometer (Thermo Fisher Scientific), and the approved samples [optical density at $260 \mathrm{~nm}(\mathrm{OD} 260)<1.7$ and OD280 $<2.0$ ] were stored at $-80^{\circ} \mathrm{C}$. Contaminating DNA was removed by DNase treatment; the RNA was digested at room temperature in $5 \times$ gDNA Eraser buffer (Takara Bio) for $5 \mathrm{~min}$, and then reverse-transcribed into cDNA using the PrimeScript cDNA RT kit (Takara Bio). The reaction mixture contained the following: $1 \mu \mathrm{g}$ RNA, $4 \mu \mathrm{l} 5 \times$ PrimeScript Buffer 2 (for real-time PCR), $1 \mu$ l PrimeScript RT Enzyme Mix I, $1 \mu$ RT Primer Mix, and RNase $\mathrm{dH}_{2} \mathrm{O}$ in a final volume of $20 \mu \mathrm{l}$. The reaction was performed on a C1000 Touch thermal cycler (Bio-Rad, Hercules, CA, USA) under the following conditions: $37^{\circ} \mathrm{C}$ for $15 \mathrm{~min}, 85^{\circ} \mathrm{C}$ for $5 \mathrm{~s}$, and $4{ }^{\circ} \mathrm{C}$ for an unlimited duration. The cDNA samples were stored at $-20^{\circ} \mathrm{C}$. 
Quantitative real-time (qRT-)PCR analysis of growth-associated protein 43 (GAP-43) mRNA expression in FC tissue and HMC3 cells

qRT-PCR was performed using SYBR R Premix Ex Taq II (Tli RNaseH Plus, 2x) (Takara Bio) on a CFX96 Real-Time PCR system (Bio-Rad) according to the manufacturer's protocol. The reaction volume was $25 \mu \mathrm{l}$. Relative gene expression levels were calculated with the comparative cycle threshold method with normalisation to the housekeeping gene glyceraldehyde 3-phosphate dehydrogenase (GAPDH). The following forward and reverse primers were used for amplification: human GAP-43, 5'-

GCTCTGAATTATGCCACCCC-3' and 5'-AGGTCGAACTGCTCTCTGAA-3'; rhesus macaque GAP-43, 5'AAGAAGCGAGAGGGTGATGC-3' and 5'-GTGGCACTTTCTGTCTCAGC-3'; human GAPDH, 5'-

TGAGAACGGGAAGCTTGTCA-3' and 5'- ATCGCCCCACTTGATTTTGG-3'; and rhesus macaque GAPDH, 5'GCACCACCAACTGCTTAGCAC-3' and 5'-TCTTCTGGTGGCAGTGATG-3'.

\section{Western blot analysis of GAP-43 protein expression in FC tissue and $\mathrm{HMC} 3$ cells}

Total protein was extracted from FC tissue and HMC3 cells using PMSF and RIPA buffer and quantified with the bicinchoninic acid method. The western V3 workflow (Bio-Rad) was used for western blot analysis. The primary antibodies [anti-GAP-43 (ab12274 and ab11136) and anti-GAPDH (ab181602)] and secondary antibody [horseradish peroxidase-conjugated goat anti-rabbit IgG H\&L (ab6721)] were purchased from Abcam (Shanghai, China).

\section{Results}

\section{Quality control for proteomics analysis}

The experimental protocol is illustrated in Fig. 1. We identified 26,493 and 26,309 peptides in 2 independent ITRAQ experiments, corresponding to 3598 and 3590 proteins, respectively. We judged whether the identified proteins were reliable based on the unique peptides, which only exist in one protein. The number of proteins containing at least 2 unique peptides in the 2 datasets was 2909 and 2918, accounting for $80.85 \%$ and $81.28 \%$ of total proteins, respectively (Fig. $2 \mathrm{~A}$ ). As each mass spectrometer has a defined measurement range, the peptides that can be identified have a length limit. The identified peptides had lengths between 7 and 20, with a mode of 11 and a mean of 13.98, which was within a reasonable range (Fig. 2B). A greater number of peptides increases the reliability of an identified protein; thus, the coverage of identified proteins indirectly reflects the overall accuracy of the results. The percentage of proteins with coverage of $0-10 \%$ was $38.49 \%$; the average protein coverage was $20.76 \%$, and proteins with coverage $\geq 20 \%$ accounted for $39.28 \%$ of total proteins (Fig. $2 \mathrm{C}$ ). These results demonstrate that our iTRAQ results were relatively reliable.

\section{Identification and function annotation of DEPs}


We used 3 different databases (GO, KEGG pathway, and COG) to predict the functions and biological significance of proteins identified by iTRAQ analysis for LNB. Not all of the 4450 identified proteins were annotated in the databases; 4321, 2339, and 2642 proteins were annotated in the GO, COG, and KEGG databases, respectively.

The identified proteins were divided into $3 \mathrm{GO}$ functional classes (Biological Process, Cellular Component, and Molecular Function), and subcategorised into 58 hierarchical GO classifications for detailed investigation (Fig. 2D-F). We performed independent functional annotation analyses on proteins that were up- and downregulated over time in FC/Bb co-cultures. For example, there were 368 DEPs (299 upregulated and 69 downregulated) in FC samples cultured with Bb as compared to PBS for $24 \mathrm{~h}$ (Bb$24 \mathrm{~h}$ and P-24 h, respectively); a comparison of $\mathrm{GO}$ annotation results revealed major differences in $\mathrm{GO}$ functional classifications between up-and downregulated proteins (Fig. 2G). For example, the function "Protein-binding transcription factor activity" was associated with the former but not the latter set of proteins. We also compared the identified proteins with the COG database, predicted the possible functions of these proteins and made classification statistics. The COG annotations for the identified proteins are shown in Fig. $2 \mathrm{H}$.

Pathway-based analyses can identify the main biochemical, metabolic, and signal transduction pathways in which a protein is involved. We performed the KEGG pathway and functional enrichment analyses of the DEPs (Fig. 2l, J). Bb infection had a marked effect on the functional profile of the FC of rhesus macaque, as evidenced by the fact that the DEPs were mainly associated with the pathogenesis of Parkinson's disease, Huntington disease, Alzheimer's disease, and signalling pathways related to neural function (Supplemental Table 1,2).

\section{Analysis of DEPs}

We analysed differences in protein expression levels between the Bb-infected and control (PBS) samples (fold change $\geq 1.5$ or $\leq 0.67, P \leq 0.05$ ) [19]. The number of DEPs between the experimental and control groups increased over time (Fig. 3A); a heatmap of the DEPs is shown in Fig. 3B, and the top 10 up- and downregulated proteins in each group are shown in Supplemental Table 3. GAP-43 level decreased over time in the PBS/Bb-treated FC explants, but the level was significantly greater in the Bb group compared with the PBS group at $24 \mathrm{~h}$ time point (fold change $=24.59, \mathrm{P}=0.012$ ).

To investigate the interactions of proteins in the FC in response to $\mathrm{Bb}$ infection, we performed a proteinprotein interaction (PPI) network analysis using the STRING web resource (https://string-preview.org/) and Cytoscape v3.8.1 software. The PPI network contained 28 nodes and 101 edges and revealed network crosstalk between GAP-43 and related DEPs (Fig. 3C). We selected GAP-43 for more detailed analysis as it was located upstream of the PPI network.

\section{Validation of GAP-43 mRNA and protein expression in FC explants stimulated with $\mathrm{Bb}$}


We examined GAP-43 mRNA and protein expression in rhesus monkey FC explants stimulated with Bb by qRT-PCR and western blot, respectively. There was no significant difference in GAP-43 protein level in FC explants infected with $\mathrm{Bb}$ for $12 \mathrm{~h}$ relative to the PBS control; however, at $24 \mathrm{~h}, \mathrm{GAP}-43$ level was higher in the $\mathrm{Bb}$ infection group (Fig. 4A, B). Additionally, GAP-43 mRNA level was higher in the Bb group than in the PBS group at 12 and $24 \mathrm{~h}$, which is consistent with the results of the proteomics analysis (Fig. $4 \mathrm{C}$ ).

\section{Validation of GAP-43 mRNA and protein expression in HMC3 cells stimulated with Bb}

We investigated whether $\mathrm{Bb}$ affects GAP-43 mRNA and protein expression in human microglia using HMC3 cells at 6, 12, and $24 \mathrm{~h}$ after infection. At all three time points, GAP-43 protein (Fig. 5A, B) and mRNA (Fig. 5C) expression was upregulated in $\mathrm{HMC} 3$ cells treated with $\mathrm{Bb}$ compared to those treated with PBS.

\section{Discussion}

LD is a natural disease caused by infection with $\mathrm{Bb}$ [20], which uses various strategies to overcome the host's innate and adaptive immune responses and induces systemic symptoms [21] including neurologic dysfunction [22]. Infection of the brain is considered as the most dangerous manifestation of LD. In order to gain insight into the mechanism by which $\mathrm{Bb}$ causes neurologic damage, we carried out proteome profiling of rhesus macaque $\mathrm{FC}$ explants infected with $\mathrm{Bb}$, and verified the findings using a human microglia cell line. Spirochetes have been detected at nerve roots in a rhesus macaque model of LNB [23]. The HMC3 cell line was established through SV40-dependent immortalisation of human embryonic microglia and was recently authenticated by the American Type Culture Collection (ATCC CRL-3304; Manassas, VA, USA), and has been used in several studies [9, 24, 25].

Our analyses demonstrated that Bb infection altered the proteomic landscape of the brain in an NHP. More specifically, GAP-43 was among many proteins that were differentially expressed between FC explants or HMC3 cells exposed to Bb vs PBS; at each examined time point, the mRNA and protein levels of GAP-43 were upregulated in the Bb-treated samples. GAP-43 is mainly expressed in the endoolfactory cortex, neocortex, and hippocampus, and is anchored on the cytoplasmic side of the presynaptic plasma membrane where it regulates synaptic plasticity and regenerative axonal growth and plays a critical role in neural development, axonal outgrowth, and stabilisation of synaptic function [26]. GAP-43 was shown to promote neurite outgrowth during development and nervous system repair by inducing F-actin accumulation, stimulating morphogenic activity, and preventing growth cone retraction [27, 28]; changes in GAP-43 expression have been reported following nerve injury and in CNS diseases such as stroke, Alzheimer disease, epilepsy, and Parkinson disease [29-33].

Like many bioactive proteins, GAP-43 is intrinsically disordered, lacking a stable tertiary and/or secondary structure under in vitro physiologic conditions $[34,35]$, the functional significance of this feature has not been thoroughly studied[36]. Nonetheless, many disordered proteins such as amyloid $\beta$-protein and tau protein are soluble or can form aggregates that have been linked to the pathogenesis of 
neurodegenerative diseases [37]. GAP-43 functions as an actin regulator and changes in its expression can lead to accumulation of the actin-depolymerising factor cofilin and the formation of cofilin-actin rods, which can impair synaptic function and cause the loss of synapses [38]. Nerve damage stimulates the release of cytokines and neurotrophic factors by neurons and glia and activates growth-related proteins such as GAP-43, thereby enhancing neuroprotection and regeneration [39,40]. Given its dual functions in the CNS, it remains unclear whether the observed upregulation of GAP-43 in LNB is pathogenic or protective.

$\mathrm{Bb}$ induces the production of inflammatory mediators in the CNS, which is accompanied by neuronal and/or glial apoptosis $[41,42]$. We previously analysed the transcriptome profile of rhesus macaques brain preFC explants following $\mathrm{Bb}$ infection; the results showed that genes related to the immune response were significantly upregulated including interleukin 6 (IL-6) signal transducer (IL6ST), colonystimulating factor 1 receptor (CSF1R), C1q and tumour necrosis factor-related 7 (C1QTNF7), CX3C chemokine receptor 1 (CX3CR1), and C-C motif chemokine ligand 24 (CCL24) [8]. While most neurons in the CNS of adult mammals cannot regenerate axons after injury, increased levels of IL- 6 can activate the phosphatidylinositol 3-kinase (PI3K)/protein kinase B (Akt) and Janus kinase (JAK)/signal transducer and activator of transcription 3 (STAT3) signalling pathways, resulting in the upregulation of GAP-43 and other growth factors and consequently, neurite outgrowth and synapse formation [43,44]. Whether this mechanism applies to the repair of nerve damage caused by LNB remains to be determined. Our PPI network analysis revealed several other proteins besides GAP-43 that were upregulated in FC explants by $\mathrm{Bb}$ infection and can potentially mediate neuronal repair including synaptophysin (SYP), brain acidsoluble protein 1 (BASP1), and synapsin-1 (SYN-1).

Given the severity of clinical symptoms and poor prognosis of LNB, there is an urgent need to establish the pathogenic mechanisms and identify predictive biomarkers and therapeutic targets. Our study demonstrated that infection of FC explants from an NHP with $\mathrm{Bb}$-the causative agent of LNB-altered the expression of many proteins including GAP-43. Although these findings are preliminary and must be verified in additional studies, they provide a basis for further exploration of the pathogenesis of LNB and indicate that therapeutic targeting of GAP-43 may be a promising strategy for its treatment.

\section{Conclusion}

Our results indicate that the expression of GAP-43 was upregulated in FC explants and HMC3 cells by Bb infection, which might be associated with the pathogenesis of LNB. In conclusion, elevated GAP-43 expression is a potential marker for LNB that may be useful for diagnosis or treatment.

\section{Abbreviations}

\section{BASP1}

brain acid-soluble protein 1; $\mathrm{Bb}=$ Borrelia burgdorferi; $\mathrm{CCL}=\mathrm{CC}$-chemokine ligand; $\mathrm{CDNA}=$ complementary deoxyribonucleic acid; CNS = nervous system; DEPs = differentially expressed proteins; $\mathrm{DTT}=$ 
Dithiothreitol; FC = frontal cortex; GAP-43 = growth-associated protein 43; GAPDH = glyceraldehyde 3phosphate dehydrogenase; IDA = Information Dependent Acquisition; IL = interleukin; iTRAQ = isobaric tags for relative and absolute quantitation; $L D=$ Lyme disease; $L N B=$ Lyme neuroborreliosis; $M E M=$ Minimal Essential Medium; mRNA = messenger ribonucleic acid; MS = Mass spectrometry; NHPs = Nonhuman primates; $\mathrm{PBS}=$ phosphate-buffered saline; $\mathrm{PMSF}$ = phenylmethane sulfonyl fluoride; RIPA = radioimmunoprecipitation assay; $\mathrm{RPMI}=$ Roswell Park Memorial Institute; SYN-1 = synapsin-1; SYP = synaptophysin; TEAB = triethylammonium bicarbonate

\section{Declarations}

\section{Acknowledgements}

The authors thank the Yunnan Province Key Laboratory for Tropical Infectious Diseases in Universities; Yunnan Province Integrative Innovation Center for Public Health, Diseases Prevention and Control; Kunming Medical University; and Yunnan Demonstration Base of International Science and Technology Cooperation for Tropical Diseases (all located in Kunming, China) for supporting this study.

\section{Authors' contributions}

FKB, AHL, LBL, LSL and TGC conceived and designed the experiments. LBL, LSL, TGC, MBM, LYT and YP developed the methodology. LBL, LSL, TGC, YZ, XX, WjC, PY, YXF, MXL and JJC performed all experiments. LBL, LSL, TGC, YD, JK, SYL, BXL, SYW and GZZ analyzed and discussed the data. LBL, LSL and TGC wrote the manuscript. LBL, LSL, TGC, FKB and AHL edited and revised the manuscript. All authors read and approved the manuscript.

\section{Funding}

This work was supported by grants from the National Natural Science Foundation of China (No. $32060180,81860644,81560596$, and 31560051) and the Natural Foundation of Yunnan Province [No. 2019FE001(-002) and 2017FE467(-001)]. The funding institutions had no involvement in the design of the study or review of the manuscript.

Availability of data and materials

All data generated or analyzed during this study are included in this published article [and its supplementary information files].

\section{Ethics approval and consent to participat}

This experiment was performed according to the Guide for the Care and Use of Laboratory Animals and ARRIVE Guidelines for Reporting Animal Research. The Animal Ethics and Welfare Committee of Kunming Medical University reviewed and approved this study. 
Not applicable.

\section{Competing interests}

The authors declare that they have no competing interests.

\section{Author details}

${ }^{1}$ Department of Microbiology and Immunology, Kunming Medical University, Kunming 650500, China;

${ }^{2}$ Department of Biochemistry and Molecular Biology, Kunming Medical University, Kunming 650500, China;

${ }^{3}$ Yunnan Province Key Laboratory of Children's Major Diseases Research, The Children's Hospital of Kunming / Kunming Medical University, Kunming 650030, China; ${ }^{4}$ The Institute for Tropical Medicine, Kunming Medical University, Kunming 650500, China; ${ }^{5}$ Yunnan Demonstration Base of International Science and Technology Cooperation for Tropical Diseases, Kunming 650500, China.

\section{Footnotes}

${ }^{\dagger}$ These authors contributed equally to the paper.

*Correspondence: liuaihua@kmmu.edu.cn(Prof A Liu), baofukai@kmmu.edu.cn(Prof F Bao)

\section{References}

1. Koedel U, Pfister HW. Lyme neuroborreliosis. Curr Opin Infect Dis. 2017;30(1):101-7.

2. Younger DS. Epidemiology of Lyme Neuroborreliosis. Neurol Clin. 2016;34(4):875-86.

3. Lalor SJ, Segal BM. Lymphoid chemokines in the CNS. J Neuroimmunol. 2010;224(1-2):56-61.

4. Garcia-Monco JC, Benach JL. Lyme Neuroborreliosis: Clinical Outcomes, Controversy, Pathogenesis, and Polymicrobial Infections. Ann Neurol. 2019;85(1):21-31.

5. Ramesh G, MacLean AG, Philipp MT. Cytokines and chemokines at the crossroads of neuroinflammation, neurodegeneration, and neuropathic pain. Mediators Inflamm. 2013;2013:480739.

6. Bransfield RC. Neuropsychiatric Lyme Borreliosis: An Overview with a Focus on a Specialty Psychiatrist's Clinical Practice. Healthcare (Basel). 2018;6(3):104.

7. Phillips KA, Bales KL, Capitanio JP, Conley A, Czoty PW, t Hart BA, Hopkins WD, Hu SL, Miller LA, Nader MA, Nathanielsz PW, Rogers J, Shively CA, Voytko ML. Why primate models matter. Am J Primatol. 2014;76(9):801-27.

8. Ding Z, Ma M, Tao L, Peng Y, Han Y, Sun L, Dai X, Ji Z, Bai R, Jian M, Chen T, Luo L, Wang F, Bi Y, Liu A, Bao F. Rhesus Brain Transcriptomic Landscape in an ex vivo Model of the Interaction of Live 
Borrelia Burgdorferi With Frontal Cortex Tissue Explants. Front Neurosci. 2019;13:651.

9. Dello Russo C, Cappoli N, Coletta I, Mezzogori D, Paciello F, Pozzoli G, Navarra P, Battaglia A. The human microglial HMC3 cell line: where do we stand? A systematic literature review. J Neuroinflammation. 2018;15(1):259.

10. Morris GP, Clark IA, Zinn R, Vissel B. Microglia: a new frontier for synaptic plasticity, learning and memory, and neurodegenerative disease research. Neurobiol Learn Mem. 2013;105:40-53.

11. Dello Russo C, Lisi L, Tentori L, Navarra P, Graziani G, Combs CK. Exploiting Microglial Functions for the Treatment of Glioblastoma. Curr Cancer Drug Targets. 2017;17(3):267-81.

12. Du L, Zhang Y, Chen Y, Zhu J, Yang Y, Zhang HL. Role of Microglia in Neurological Disorders and Their Potentials as a Therapeutic Target. Mol Neurobiol. 2017;54(10):7567-84.

13. Kaur U, Meng H, Lui F, Ma R, Ogburn RN, Johnson JHR, Fitzgerald MC, Jones LM. Proteome-Wide Structural Biology: An Emerging Field for the Structural Analysis of Proteins on the Proteomic Scale. J Proteome Res. 2018;17(11):3614-27.

14. Liu CM, Chen J, Yang S, Jiang TT, Chen ZL, Tu HH, Mao LG, Hu YT, Gan L, Li ZJ, Li JC. iTRAQ-based proteomic analysis to identify the molecular mechanism of Zhibai Dihuang Granule in the Yindeficiency-heat syndrome rats. Chin Med. 2018;13:2.

15. Savage CR, Jutras BL, Bestor A, Tilly K, Rosa PA, Tourand Y, Stewart PE, Brissette CA, Stevenson B. Borrelia burgdorferi SpoVG DNA- and RNA-Binding Protein Modulates the Physiology of the Lyme Disease Spirochete. J Bacteriol. 2018;200(12):e00033-18.

16. Peng M, Li S, He Q, Zhao J, Li L, Ma H. Proteomics reveals changes in hepatic proteins during chicken embryonic development: an alternative model to study human obesity. BMC Genom. 2018;19(1):29.

17. Zückert WR. Laboratory Maintenance of Borrelia burgdorferi. Curr Protoc Microbiol. 2007;4(1):12C.1.1-C.1.0.

18. Ruzić-Sabljić E, Lotric-Furlan S, Maraspin V, Cimperman J, Logar M, Jurca T, Strle F. Comparison of isolation rate of Borrelia burgdorferi sensu lato in MKP and BSK-II medium. Int J Med Microbiol. 2006;296 Suppl 40:267 - 73.

19. Liu F, Xiong Y, Zhao Y, Tao L, Zhang Z, Zhang H, Liu Y, Feng G, Li B, He L, Ma J, Qin S, Yang Y. Identification of aberrant microRNA expression pattern in pediatric gliomas by microarray. Diagn Pathol. 2013;8:158.

20. Shapiro ED. Clinical practice. Lyme disease. N Engl J Med. 2014;370(18):1724-31.

21. de Taeye SW, Kreuk L, van Dam AP, Hovius JW, Schuijt TJ. Complement evasion by Borrelia burgdorferi: it takes three to tango. Trends Parasitol. 2013;29(3):119-28.

22. Rupprecht TA, Koedel U, Fingerle V, Pfister HW. The pathogenesis of lyme neuroborreliosis: from infection to inflammation. Mol Med. 2008;14(3-4):205-12.

23. Pachner AR, Gelderblom H, Cadavid D. The rhesus model of Lyme neuroborreliosis. Immunol Rev. 2001;183:186-204. 
24. Angel-Ambrocio AH, Bautista-Carbajal P, García-León ML, Gomora-Herrera MJ, Pedernera-Astegiano EA, Wong-Chew RM. Microglia HMC3 cells are highly susceptible to Rhinovirus infection. Virus Res. 2020;288:198110.

25. Hankittichai P, Lou HJ, Wikan N, Smith DR, Potikanond S, Nimlamool W. Oxyresveratrol Inhibits IL-1 $\beta$ Induced Inflammation via Suppressing AKT and ERK1/2 Activation in Human Microglia, HMC3. Int J Mol Sci. 2020;21(17):6054.

26. Holahan MR. A Shift from a Pivotal to Supporting Role for the Growth-Associated Protein (GAP-43) in the Coordination of Axonal Structural and Functional Plasticity. Front Cell Neurosci. 2017;11:266.

27. Camporesi E, Nilsson J, Brinkmalm A, Becker B, Ashton NJ, Blennow K, Zetterberg H. Fluid Biomarkers for Synaptic Dysfunction and Loss. Biomark Insights. 2020;15:1177271920950319.

28. Zhang F, Ying L, Jin J, Feng J, Chen K, Huang M, Wu Y, Yu H, Su D. GAP43, a novel metastasis promoter in non-small cell lung cancer. J Transl Med. 2018;16(1):310.

29. Nemes AD, Ayasoufi K, Ying Z, Zhou QG, Suh H, Najm IM. Growth Associated Protein 43 (GAP-43) as a Novel Target for the Diagnosis, Treatment and Prevention of Epileptogenesis. Sci Rep. 2017;7(1):17702.

30. Sandelius Å, Cullen NC, Källén Å, Rosengren L, Jensen C, Kostanjevecki V, Vandijck M, Zetterberg H, Blennow K. Transient increase in CSF GAP-43 concentration after ischemic stroke. BMC Neurol. 2018;18(1):202.

31. Sandelius Å, Portelius E, Källén Å, Zetterberg H, Rot U, Olsson B, Toledo JB, Shaw LM, Lee VMY, Irwin DJ, Grossman M, Weintraub D, Chen-Plotkin A, Wolk DA, McCluskey L, Elman L, Kostanjevecki V, Vandijck M, McBride J, Trojanowski JQ, Blennow K. Elevated CSF GAP-43 is Alzheimer's disease specific and associated with tau and amyloid pathology. Alzheimers Dement. 2019;15(1):55-64.

32. Tible M, Sandelius Å, Höglund K, Brinkmalm A, Cognat E, Dumurgier J, Zetterberg H, Hugon J, Paquet C, Blennow K. Dissection of synaptic pathways through the CSF biomarkers for predicting Alzheimer disease. Neurology. 2020;95(8):e953-e61.

33. Tönges L, Szegö EM, Hause P, Saal KA, Tatenhorst L, Koch JC, Z DH, Dambeck V, Kügler S, Dohm CP, Bähr M, Lingor P. Alpha-synuclein mutations impair axonal regeneration in models of Parkinson's disease. Front Aging Neurosci. 2014;6:239.

34. Forsova OS, Zakharov VV. High-order oligomers of intrinsically disordered brain proteins BASP1 and GAP-43 preserve the structural disorder. Febs j. 2016;283(8):1550-69.

35. Uversky VN. Targeting intrinsically disordered proteins in neurodegenerative and protein dysfunction diseases: another illustration of the D(2) concept. Expert Rev Proteomics. 2010;7(4):543-64.

36. Chiti F, Dobson CM. Protein misfolding, functional amyloid, and human disease. Annu Rev Biochem. 2006;75:333-66.

37. Caminati G, Procacci P. Mounting evidence of FKBP12 implication in neurodegeneration. Neural Regen Res. 2020;15(12):2195-202.

38. Chung D, Shum A, Caraveo G. GAP-43 and BASP1 in Axon Regeneration: Implications for the Treatment of Neurodegenerative Diseases. Front Cell Dev Biol. 2020;8:567537. 
39. Filbin MT. Myelin-associated inhibitors of axonal regeneration in the adult mammalian CNS. Nat Rev Neurosci. 2003;4(9):703-13.

40. Silver J, Miller JH. Regeneration beyond the glial scar. Nat Rev Neurosci. 2004;5(2):146-56.

41. Ramesh G, Borda JT, Dufour J, Kaushal D, Ramamoorthy R, Lackner AA, Philipp MT. Interaction of the Lyme disease spirochete Borrelia burgdorferi with brain parenchyma elicits inflammatory mediators from glial cells as well as glial and neuronal apoptosis. Am J Pathol. 2008;173(5):1415-27.

42. Ramesh G, Didier PJ, England JD, Santana-Gould L, Doyle-Meyers LA, Martin DS, Jacobs MB, Philipp MT. Inflammation in the pathogenesis of lyme neuroborreliosis. Am J Pathol. 2015;185(5):1344-60.

43. Kossmann T, Hans V, Imhof HG, Trentz O, Morganti-Kossmann MC. Interleukin-6 released in human cerebrospinal fluid following traumatic brain injury may trigger nerve growth factor production in astrocytes. Brain Res. 1996;713(1-2):143-52.

44. Yang P, Wen H, Ou S, Cui J, Fan D. IL-6 promotes regeneration and functional recovery after cortical spinal tract injury by reactivating intrinsic growth program of neurons and enhancing synapse formation. Exp Neurol. 2012;236(1):19-27.

\section{Figures}

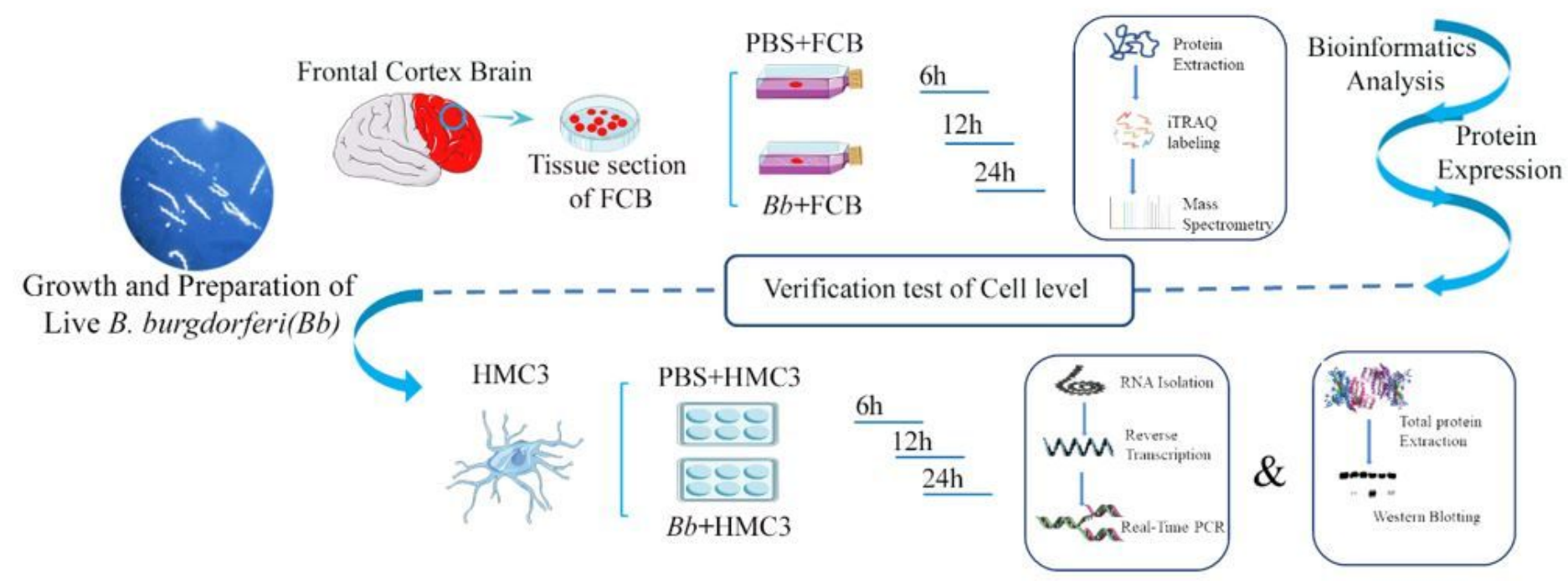

\section{Figure 1}

Schematic illustration of the experimental design. 
A

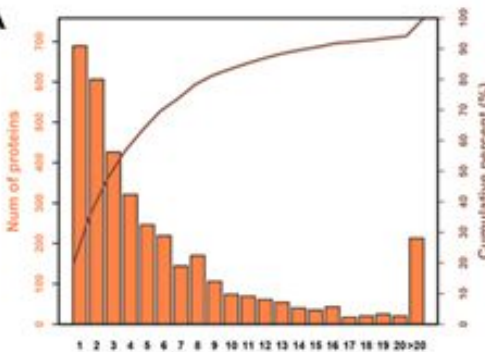

Num of unique peptides

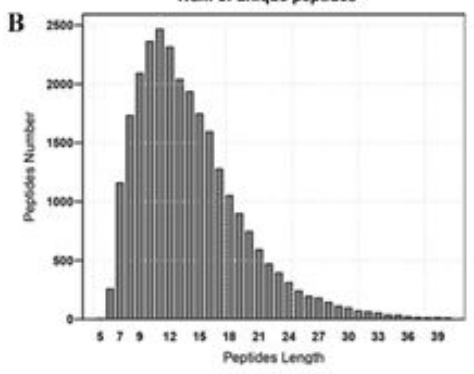

C

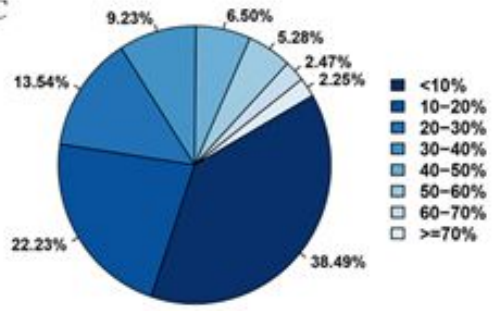

D

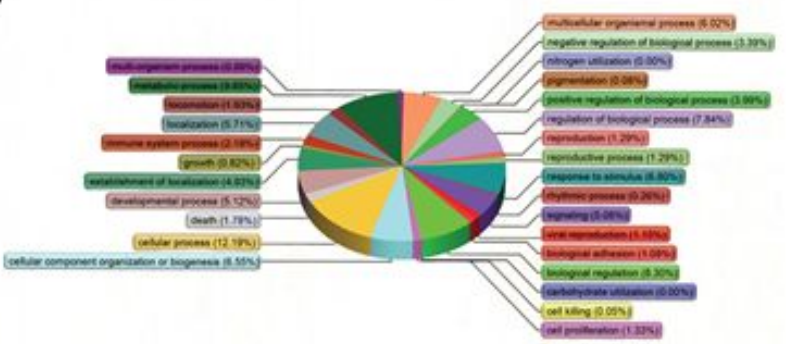

E

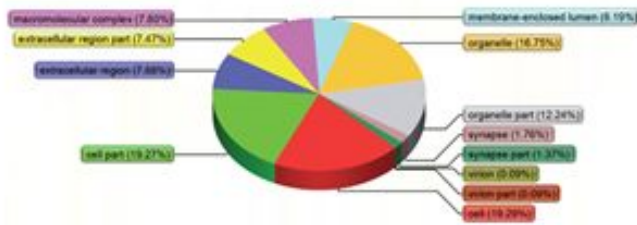

$\mathbf{F}$

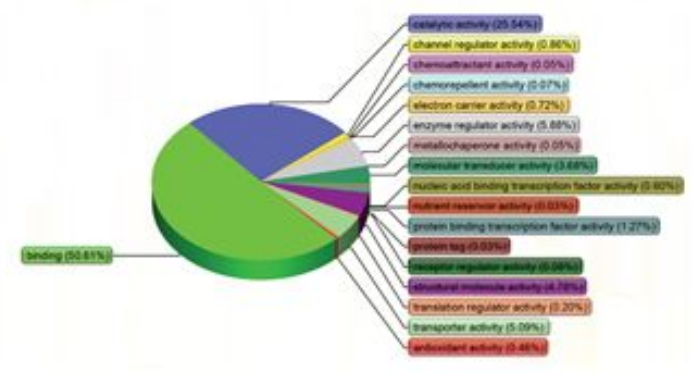

G
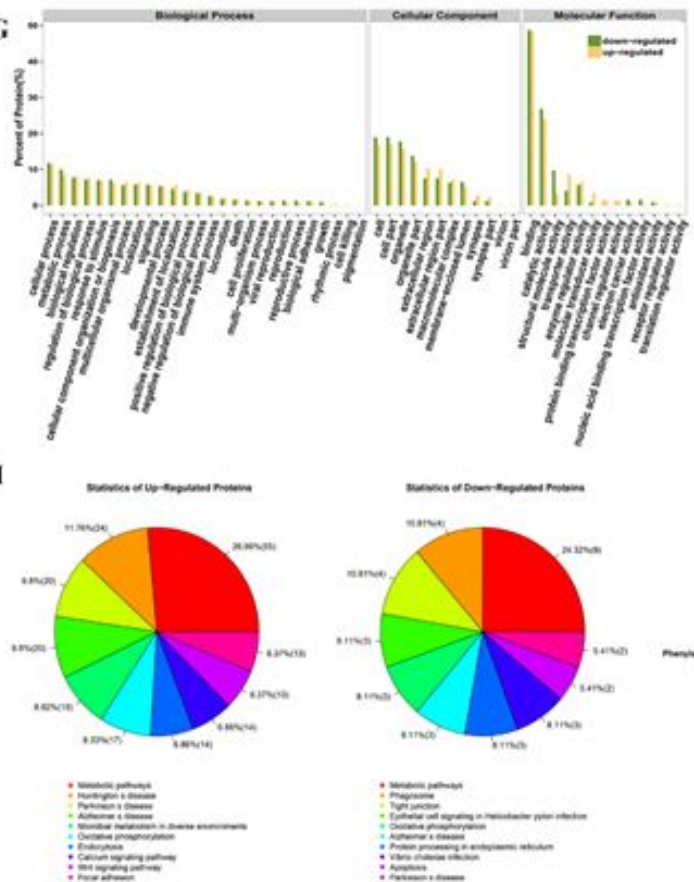

H
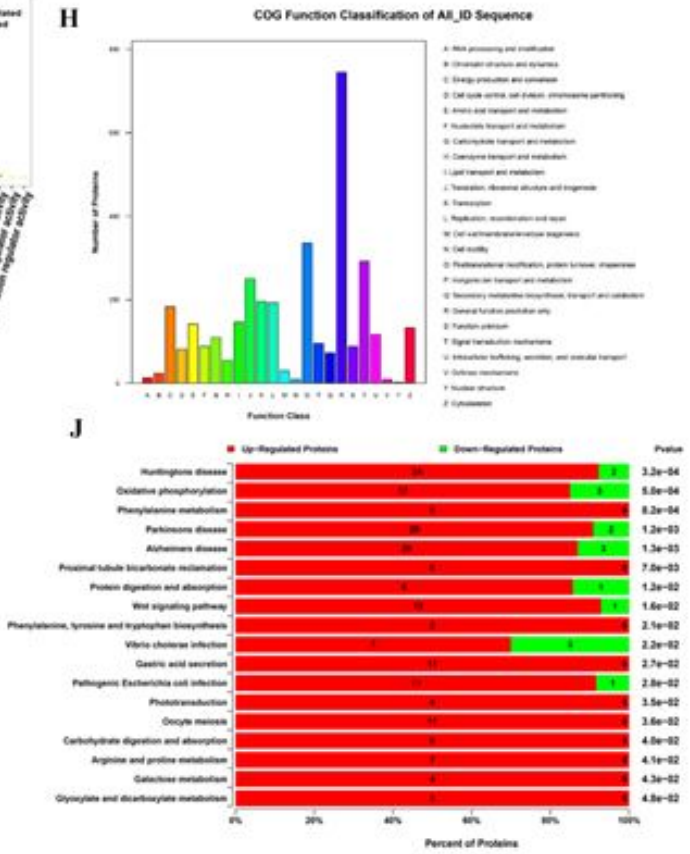

Figure 2

Quality control of proteomics analysis and quantification of DEPs. A. Number of unique peptides of the identified proteins. B. Peptides length. C. Coverage of identified proteins. D-F. GO classification of all quantified proteins in the domains of Biological Process, Cellular Component, and Molecular Function. G. $\mathrm{GO}$ classification of DEPs in the 3 domains in the Bb-24h/P-24h group. H. COG functional classification 
of all quantified proteins. I. Up- and downregulated protein pathways in the Bb-24h/P-24h group. J. Pathway analysis of DEPs in the Bb-24h/P-24h group.
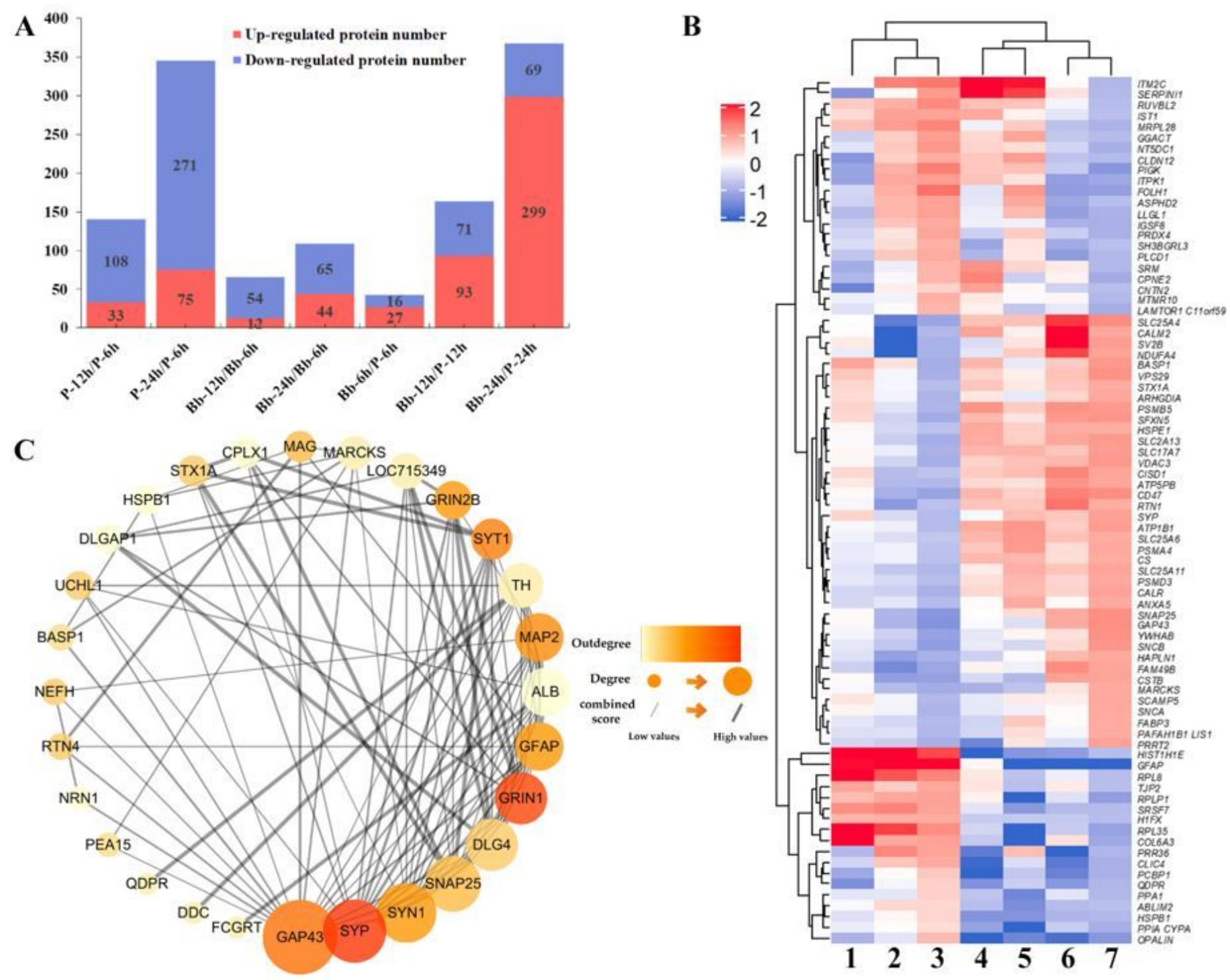

Figure 3

Screening of DEPs. A. Number of DEPs in different groups. B. Heatmap of proteins with significantly different expression in each group (1, Bb-6h/PBS-6h; 2, PBS-12h/PBS-6h; 3, PBS-24h/PBS-6h; 4, Bb-12h/ Bb-6h; 5, Bb-24h/ Bb-6h; 6, Bb-12h/PBS-12h; 7, Bb-24h/PBS-24h). C. Analysis of the GAP-43 PPI network. 
A
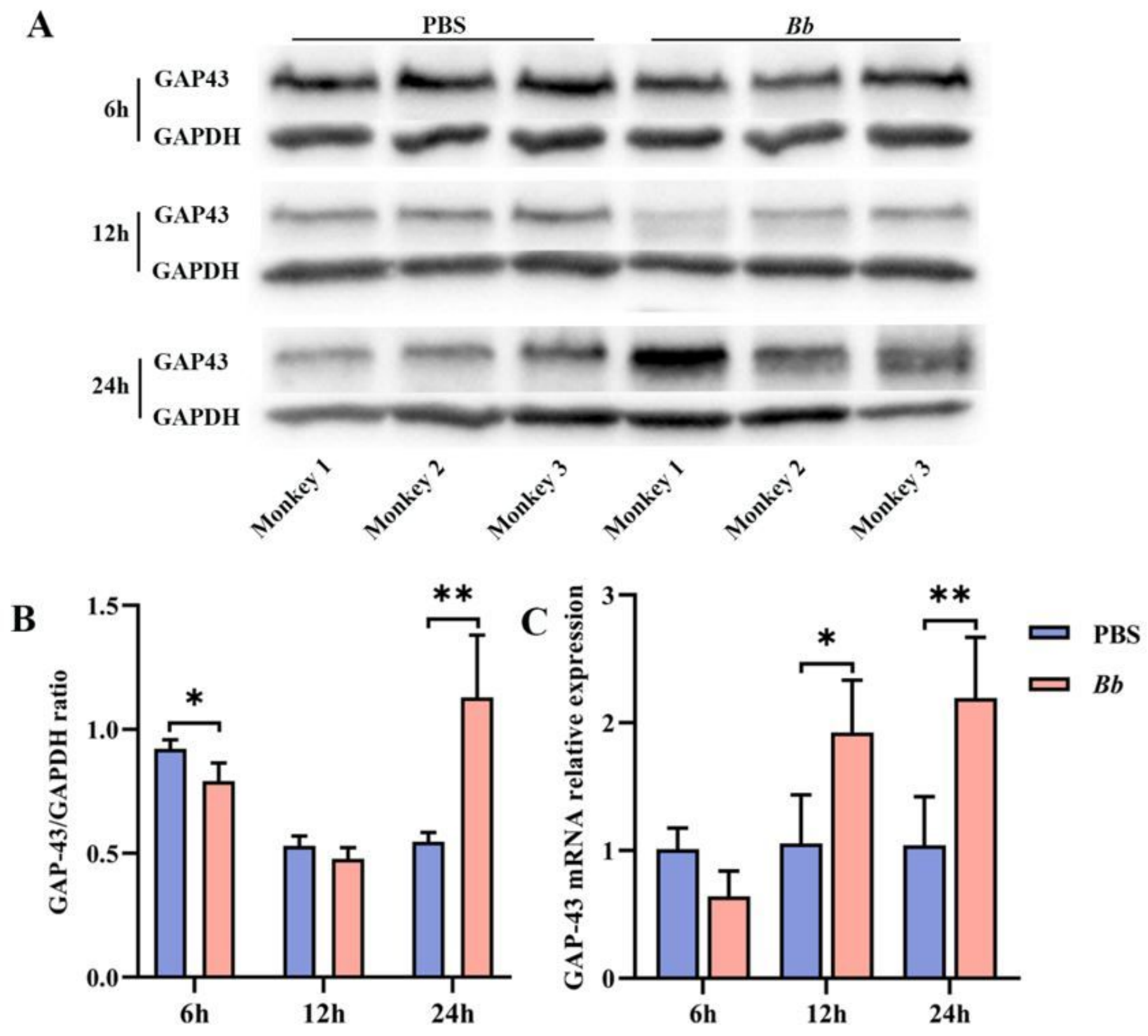

Figure 4

GAP-43 mRNA and protein levels in FC of rhesus macaque at different time points after Bb infection. A. Representative immunoblots of GAP-43 expression at indicated time points after infection. B,C. Quantitative analysis of GAP-43 protein (B) and relative mRNA (C) expression levels. 
A

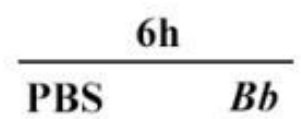

\begin{tabular}{c}
\multicolumn{2}{c}{$12 \mathrm{~h}$} \\
\hline PBS $\quad B b$
\end{tabular}

\begin{tabular}{c} 
24h \\
\hline PBS $\quad B b$
\end{tabular}

GAP-43

GAPDH
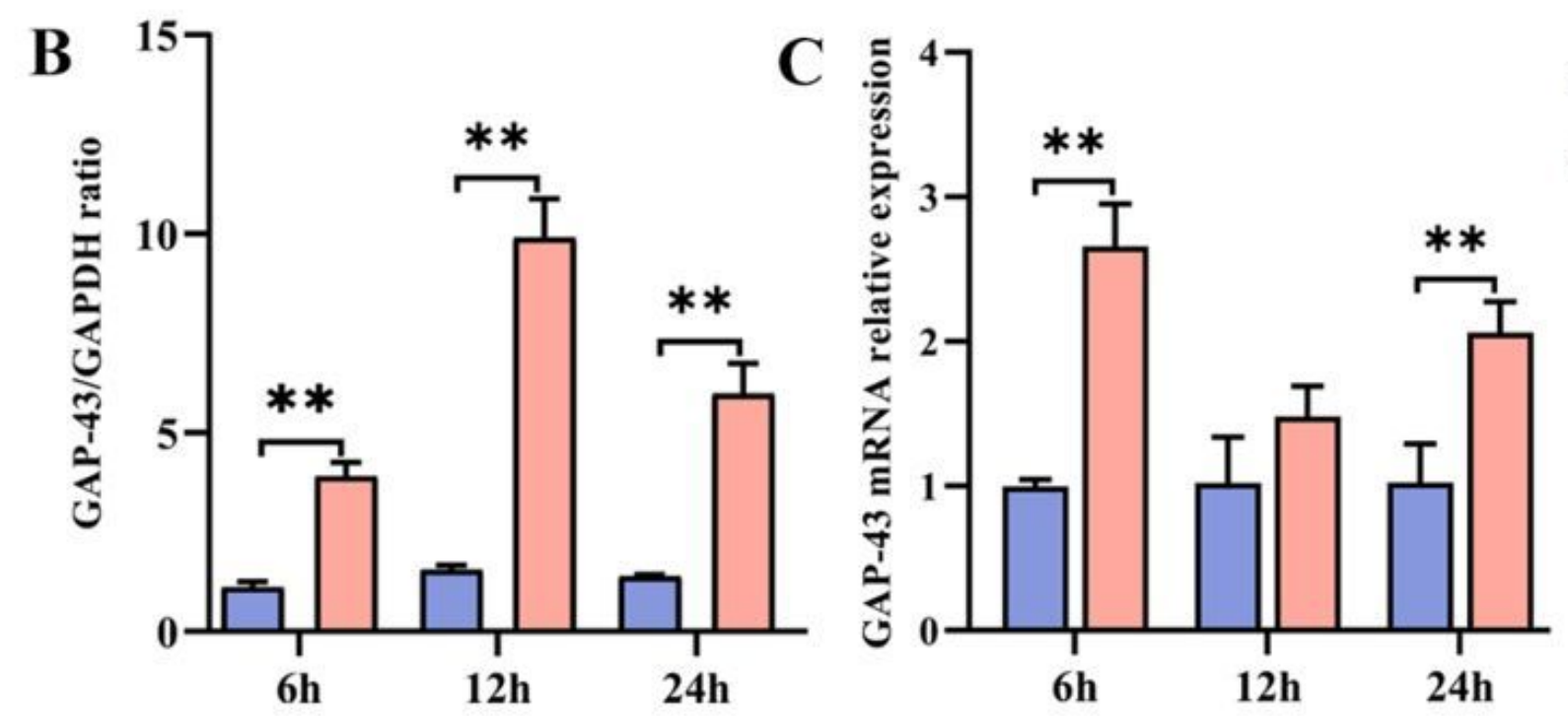

Figure 5

GAP-43 mRNA and protein levels at different time points after Bb infection with HMC3. A. Representative immunoblots of GAP-43 expression at indicated time points after infection. B,C. Quantitative analysis of GAP-43 protein (B) and relative mRNA (C) expression levels.

\section{Supplementary Files}

This is a list of supplementary files associated with this preprint. Click to download.

- SupplementalTable1GOenrichmentofDEPs.xIsx

- SupplementalTable2PathwayenrichmentofDEPs.xlsx

- SupplementalTable3ScreeningofDEPs.xlsx 